\title{
A STUDY ON THE DYNAMIC UPLIFTING OF FLEXIBLE STRUCTURE ON COMPLIANT GROUND
}

\author{
By Benito PACHECO*, Yozo FUJINO** and Manabu ITO***
}

\begin{abstract}
A relatively simple 5-DOF model is used to demonstrate the nature of partial uplift in the case of medium-frequency structure-soil system. A global-level displacement-type criterion of uplift is introduced together with a concept of instantaneous effective radius. Under the action of severe yet possible ground motions (horizontal and vertical accelerations), it is demonstrated that uplifting tends to be triggered by large input pulses. Structure-soil interaction at full-contact condition proves capable of preventing or controlling uplift, however. When it occurs, uplift reduces structural stresses but increases soil pressures and rigid-body displacements. While some finite-element models are available that are analytically more refined, the present simple model may be well suited to the evaluation of uplift effects-both positive and negative-on preliminary design configurations.

Keyword: Partial uplift ; Structure-soil interaction; Viscoelastic foundation ; Instantaneous effective radius
\end{abstract}

\section{INTRODUCTION}

A structure is said to uplift when part of its base intermittently separates vertically from the ground. While it is true that the displacements involved may be too small to be directly observable, some recent finite-element formulations, e.g. Refs. 1 and 2, can demonstrate that stress- or strain limits are occasionally reached at interface- or joint elements, thereby making any assumption of constant contact untenable. Thus, geometric nonlinearity is introduced at the interface, causing the dynamic response analysis to become quite complicated.

There is a need, however, for simplified models that can be used economically in evaluating the response of preliminary design configurations. Therefore in the present problem, as in constant-contact interaction, it is deemed useful to approximate as rigid the base which comes in contact with the soil, and use discrete springs and dashpots to model the soil continuum. In this manner the number of degrees of freedom is greatly reduced.

The recent studies reported in Refs. 3-5 are in the latter spirit. Great reductions in the base shear have been reported, particularly for short-period structures ${ }^{4)}$. Precisely because short-period structures are thought to be more likely to uplift - and uplift by greater amounts - the partial uplift phenomenon has been studied more frequently in relation to nuclear power plants $^{(6)}$-9) ; the rigid-basemat approximation is quite accurate for such cases.

The present paper also uses a global approach, in contrast with element approach in FEM. A global-level, displacement-type criterion for partial uplift is introduced, together with a simple concept of

* Graduate Student, Dept. of Civil Eng., University of Tokyo

** Member of JSCE, Ph. D., Assoc. Professor of Civil Eng., University of Tokyo

*** Member of JSCE, Dr. Eng., Professor of Civil Eng., University of Tokyo 
continually varying effective base radius. Springs and dashpots at the base are approximated from models of viscoelastic continuum $\left.{ }^{10)} \sim 11\right)$. In this paper, both the advantageous and adverse effects of partial uplift are analyzed, under the action of horizontal and vertical ground accelerations. Also explored is the possibility of predicting uplift without actual time history computation.

Sliding of the base is not considered in the present analysis. This is in contrast with finite element analyses wherein new non-linear elements are proving to be capable of predicting local sliding as well as separation ${ }^{1 \sim 2)}$. Considered here, however, is base swaying, which is disregarded in Refs. 3-5. Both swaying and sliding may be significant in the case of squat structures.

\section{ANALYTICAL MODEL AND GOVERNING EQUATIONS}

A uniform linear elastic beam-column is assumed to support a top mass and stand at the center of a rigid base slab whose own mass is nonzero (This model may be reduced to the conventional SDOF oscillator by taking the top mass to be proportionately large and neglecting rotary inertia. To model a building core or a medium-rise chimney, on the other hand, this top mass may be taken as zero instead). In turn the rigid footing is considered to rest, by gravity, on a linear viscoelastic continuum. This system and its associated properties are described in Fig. 1.

Four structures, namely, A, B, C, and D and their corresponding values of nondimensionalized parameters are described in Table 1. The present examples are basically medium-proportion structures $(L / R=4)$ of either steel $\left(E / m=25 \times 10^{6} \mathrm{sec}^{-2}\right)$ or concrete $\left(E / m=5 \times 10^{6} \mathrm{sec}^{-2}\right)$ construction. The structural damping value $\zeta$ assigned to all cases is small, as would be common of slender, towerlike

Table 1 Values of nondimensionalized parameters for the examples.

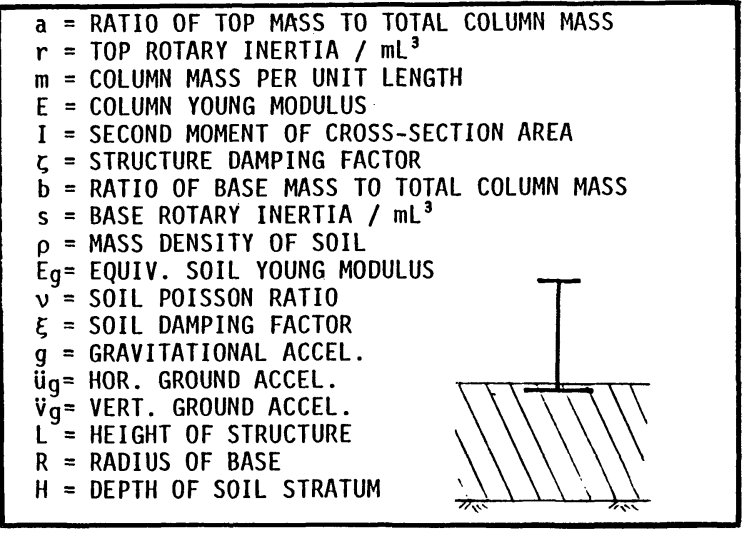

Fig.1 Definition of physical system.

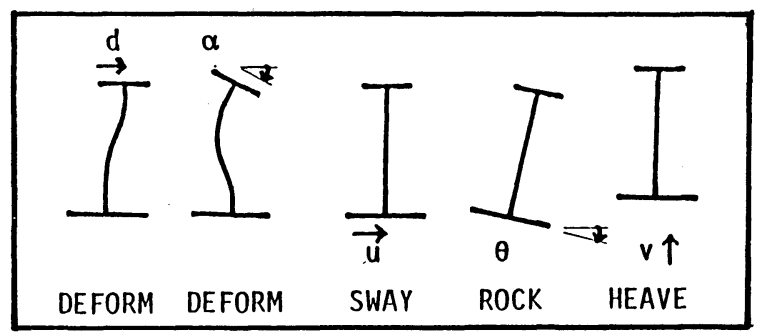

Fig. 2 Generalized displacements.

\begin{tabular}{|c|c|c|c|c|}
\hline System & A & B & C & D \\
\hline $\mathrm{E} / \mathrm{m}\left(\mathrm{s}^{-2}\right) 10^{6}$ & 5.0 & 5.0 & 25.0 & 25.0 \\
\hline$I / L^{4}$ & 1.0 & 1.0 & 2.0 & 2.0 \\
\hline$a$ & 0.5 & 0.5 & 1.0 & 1.0 \\
\hline $10^{-3}$ & 10.0 & 10.0 & 20.0 & 20.0 \\
\hline$E / E_{g}$ & 3.0 & 0.6 & 10.0 & 2.0 \\
\hline \multicolumn{2}{|c|}{$A, B, C, D$} & \multicolumn{2}{|l|}{$\xi$} & 0.10 \\
\hline b & 2.0 & $v$ & & 0.33 \\
\hline $10^{-3}$ & 50.0 & $L / R$ & & 4.0 \\
\hline$\zeta$ & 0.01 & $m / \pi R^{2} \rho$ & $10^{-3}$ & 10.0 \\
\hline$R / H$ & 0.0 & $g \rho R / E_{g}$ & $10^{-3}$ & 5.0 \\
\hline
\end{tabular}

Table 2 Fundamental frequencies and critical damping ratios at full-contact interaction.

\begin{tabular}{|c|c|c|c|c|}
\hline System & A & $\mathrm{B}$ & C & D \\
\hline \multicolumn{5}{|c|}{ Fundamental freq., hz } \\
\hline$f_{f}$, fixed base & 0.72 & 0.72 & 1.76 & 1.76 \\
\hline$f_{i}$, interaction & 0.58 & 0.67 & 0.82 & 1.35 \\
\hline $\mathbf{f}_{f} / \mathbf{f}_{i}$ & 1.24 & 1.07 & 2.05 & 1.30 \\
\hline \multicolumn{5}{|l|}{ Damping ratio, $\%$} \\
\hline$\zeta$, structural & 1.0 & 1.0 & 1.0 & 1.0 \\
\hline $\mathrm{D}_{i}$, interaction & 6.7 & 1.9 & 16.5 & 6.6 \\
\hline$D_{i}-\boldsymbol{\zeta}$ & 5.7 & 0.9 & 15.5 & 5.6 \\
\hline \multicolumn{5}{|c|}{ Nondimensional freq. (Eq.1) } \\
\hline$a_{0}$ & 0.82 & 0.42 & 0.95 & 0.70 \\
\hline
\end{tabular}


structures. Dissipation in the ground shall also contribute to the total damping, by an amount that depends on the extent of interaction. (See Table 2).

The rigid footing is allowed to sway $(u)$ relative to the ground, heave $(v)$ and rock $(\theta)$, while the superstructure is allowed to deform essentially as a Bernoulli-Euler beam with distributed axial force. This latter deformation is associated with the tip displacement, $d$, and tip rotation, $\alpha$ (See Fig. 2). The equations of motion are given in full in the Appendix.

As the Appendix shows, there are two sources of geometric nonlinearity in the equations of motion, namely the parametric excitation due to vertical ground acceleration as it affects the stiffness of the superstructure, and the last three diagonal elements of both damping and stiffness matrices as they depend on, among other things, the contact area between footing and soil. However in the course of a numerical solution by, say, Wilson- $\theta$ step-by-step method, an instantaneous linear system may be assumed at each time step. An effective base radius may be defined whenever there is partial uplifting.

The spring and dashpot coefficients at the base are approximated from Refs. 10 and 11 . The frequency-dependent dynamic impedances in these reports are, of course, rigorously applicable only to steady-state harmonic vibration. For transient response analysis of linear systems, this frequencydependence is commonly taken into full consideration in the frequency-domain solution prior to an inverse Fourier transformation.

Some studies, e. g. Ref. 12, have shown that it is a good approximation to take the dynamic impedance to be constant at the level corresponding to the fundamental natural frequency of the interacting system. While this scheme actually requires iteration before the representative dynamic impedance can be determined, it makes time-domain direct-integration algorithms applicable again. Thus in the present problem, which is to be solved by a direct time-integration scheme, the idea of representative dynamic impedance is being extended to a system with time-varying "fundamental" frequency. The fundamental frequency of the instantaneous system is taken as the relevant frequency $\omega$. This and the widely used nondimensionalized frequency $a_{0}$ are related in the following manner :

$$
a_{0}=\frac{\omega}{\sqrt{E / m}} \sqrt{\frac{2(1+\nu) E / E_{g}}{\pi\left(m / \pi R^{2} \rho\right)}}\left(\frac{R^{\prime}}{R}\right)
$$

where $R^{\prime}$ is the instantaneous effective radius. This iterative adjustment of ground compliance coefficients is done at the beginning of each time step. Table 2 shows the values for the present examples at full-contact condition $\left(R / R^{\prime}=1.0\right)$, together with some measures of structure-soil interaction. As may be noted, in the range of $a_{0}$ involved, lower values of $a_{0}$ generally mean less interaction.

From the generalized displacements, the dynamic internal forces may by computed. A method similar to that suggested in Ref. 13 is used here, whereby a pattern of equivalent inertial loads is associated with each mode of deformation. Such patterns of "loads" are shown in Fig. 3(a). As suggested in Fig. 3(b), in

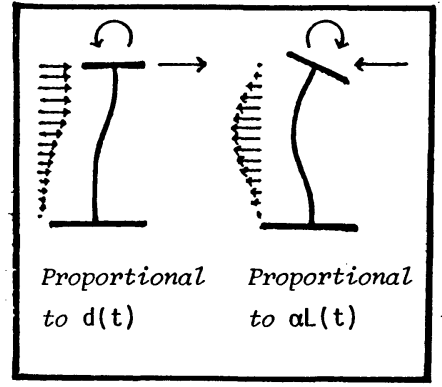

(a)

Fig. 3 Equivalent inertial loads (a) and the superposed loads (b) for obtaining base shear and moment and toe pressure.

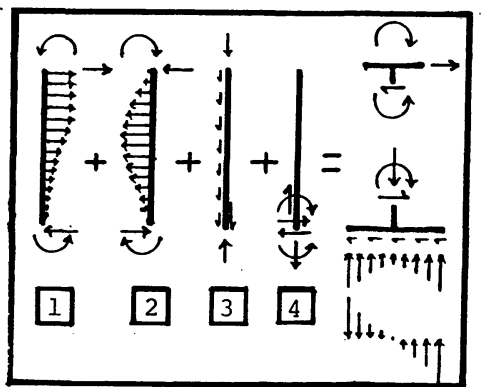

(b)

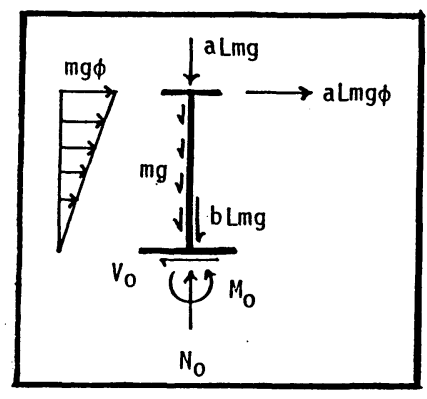

Fig. 4 Pseudostatic "design" forces. 
principle these may be superimposed with gravity force (marked 3) and additional spring forces beneath the base (marked 4]); in fact only the inertial forces 10 and 2 contribute to the transverse shear and bending moment in the tower. This scheme of internal force computation avoids much of the inaccuracy that may result from using spatial derivatives of the constraint shape functions, which were intended for use primarily in setting up generalized stiffness and mass matrices. As for the soil bearing pressure, the relevant overturning moment and normal force may be obtained directly from the product of the relevant spring stiffnesses and their corresponding deformations (actually $\theta$ and $v$ ).

The dynamic bending moment, shear, and toe pressure themselves are conveniently normalized in terms of $M_{0}, \quad V_{0}$ and $N_{0}$ :

$$
M_{0}=(1 / 3+a) L^{2} m g \phi ; V_{0}=(1 / 2+a) L m g \phi ; N_{0}=(1+a+b) L m g
$$

which would be the reactions beneath the base if the structure were to be loaded with the set of pseudostatic "design" forces as shown in Fig. 4. The "seismic load coefficient", $\phi$, is assigned a value of 0.3 in the examples in this paper.

\section{CRITERION OF PARTIAL UPLIFT AND CONCEPT OF EFFECTIVE RADIUS}

\section{(1) Critical base rotation}

Because the soil medium is flexible and there is some vertical force attributed to gravity and vertical inertia, the base (and structure) as a whole has an instantaneous "sink" amounting to $v_{t}$, which is the sum of static component $v_{s}$ and dynamic component $v$. Depending on this sink and the effective radius $R^{\prime}$ of the base, uplift (or further uplift) may be said to impend when the base rotation is such that the base heel is just about to overcome the sink. Denoting as $\theta_{c r}$ this critical base rotation for the instantaneous system, the condition of uplift (or further uplift) may be expressed as (from Fig.5) :

$$
\theta_{c r}=\frac{\left|v_{t}\right|}{R^{\prime}}
$$

on the assumption that $v_{t}<0$, i. e. , the instantaneous vertical relative displacement of the base is indeed a sink (downward). Thus the critical base rotation may be defined at the start of, and be assumed to apply to the whole duration of, each time step.

\section{( 2 ) Base rotation exceeds instantaneous critical}

At the beginning of any time step $n$, the effective radius $R^{\prime}$ and critical base rotation $\theta_{c r}$ are defined. At the end of the time step, should the computed base rotation $\theta$ exceed the critical, $R^{\prime}$ will have to be reduced (or reduced further) to account for uplift, before proceeding to the next time step. In as much as uplift at the base heel and soil overstress (hence "deterioration") at the base toe occur together, it may be enough to consider the base to "shrink" without shifting the centroid of the contact area from its initial position directly beneath the centerline of the superstructure. The reduced effective radius is merely computed such that the associated area is equal to the presumed contact surface under the partially uplifted footing (of the system at the start of the time step) (See Fig. 6). This already implies an averaging of soil bearing pressures. To be exact, one would have to define the nonuniform pressure distribution under the uplifted

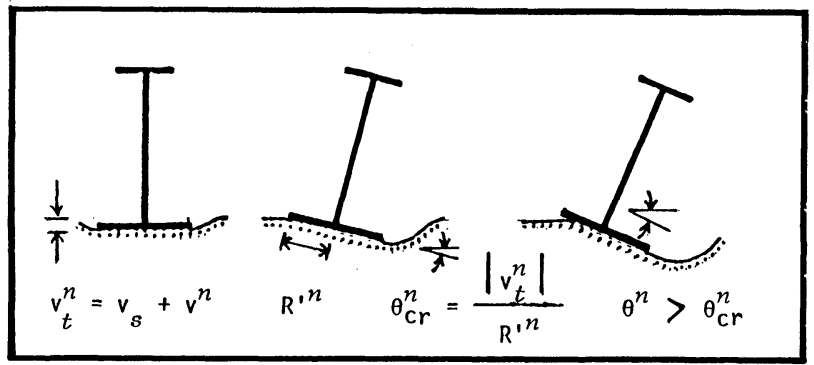

Fig. 5 Uplift criterion as applied to time step $n$.

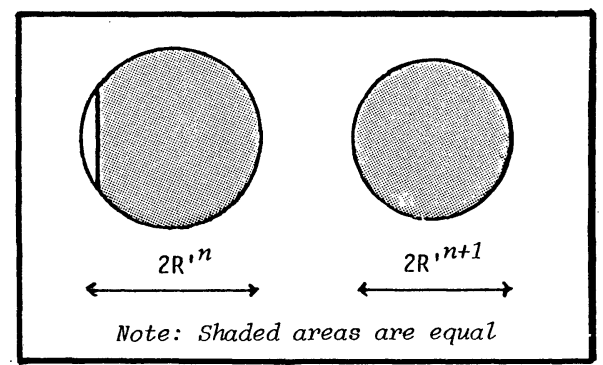

Fig. 6 Reduction of effective radius. 
base before any equation of equivalence of net moments could lead to a corresponding equation of equivalence of areas. However, having to go into such details of contact pressure for every irregular area of contact would defeat the purpose of the present simplification. Even the apparent refinement in Ref. 7, i. e. defining a different effective radius for each base mode, is at the expense of implying contadictory patterns of pressure distribution. Hence the adoption in this paper of a more straightforward approximation. The new effective radius would be used at the next time interval.

The simplest way of obtaining an effective radius for the next time step $n+1$ would be (from Fig. 6) :

$$
R^{\prime n+1}=R^{\prime n} \sqrt{\left[\pi-\cos ^{-1}\left(\frac{\theta_{c r}^{n}}{\theta^{n}}\right)+\frac{1}{2} \sin \left\{2 \cos ^{-1}\left(\frac{\theta_{c r}^{n}}{\theta^{n}}\right)\right\}\right] / \pi}
$$

\section{(3) Base rotation less than critical}

At the end of any particular time step it is possible that the computed base rotation $\theta$ is less than the instantaneous critical, indicating that uplift is not growing. Instead, when the current effective radius is less than the full radius $R$, a recovery of contact must be expressed by increasing $R^{\prime}$. The simplest scheme would be to assume that $\theta^{n}$ is equal to the critical base rotation of the next time step :

$$
R^{\prime n+1}=\frac{\left|v_{t}^{n+1}\right|}{\theta_{c r}^{n+1}} \fallingdotseq \frac{\left|v_{t}^{n}\right|}{\left|\theta^{n}\right|}, R^{\prime n+1} \leq R
$$

When the criterion discussed above in (1) is applied together with the updating schemes in (2) and (3), it is essential that the chosen time step be sufficiently small. In each example in this paper, when the time step was made approximately $1 / 10$ of the smallest natural period of the system in full-contact interaction, it was found that further reducing this time step made little difference in the integrated time history of response.

\section{(4) Comparison with other criteria}

The uplift criterion described above (Eq.3) may be classified as a displacement-type criterion on the global level. Similar criteria were used by Yim and Chopra ${ }^{3)}$ and Psycharis ${ }^{5)}$, applied to rectangular footings on Winkler foundation or two-spring foundation, respectively.

Wolf and Skrikerud ${ }^{7}$ applied what may be called a global-level force-type criterion, for circular footings on elastic halfspace. Uplift was considered initiated when the base (overturning) moment, $M_{B}$, and the total normal (vertical) force beneath the base $N_{B}$, attained the following relation :

$$
\left|\frac{M_{B}}{N_{B} \cdot R}\right|_{c r}=\frac{1}{3}
$$

As used in Ref. 7, Eq. 6 is a dynamic criterion applied at each time step, such that the effective radii during uplifting are pseudostatic functions of actual $\left|M_{B} / N_{B} R\right|$.

Eq. 6 provides a comparison in type with Eq. 3 ; moreover, it suggests a possible rewriting of Eq. 3 which leads to an expression for "critical seismic load coefficient", as outlined here next. Under the assumption now of pseudostatic conditions, Eq. 3 may be rewritten as:

$$
\left|\frac{M_{B}}{N_{B} \cdot R}\right|_{c r}=\frac{2}{3} \frac{(1+1 / 6 R / H)}{(1+1.28 R / H)}
$$

Assuming further that the base moment and the base normal force always have the static ratio of (from Fig. 4)

$$
\left|\frac{M_{B}}{N_{B} \cdot R}\right|_{\text {stat }}=\frac{(1 / 3+a)}{(1+a+b)} \frac{L}{R} \phi .
$$

then an approximate indicator of initial uplift may be obtained as in Eq. 9 below.

$$
\phi_{c r}=\frac{2}{3} \frac{(1+1 / 6 R / H)(1+a+b)}{(1+1.28 R / H)(1 / 3+a) L / R}
$$

This "critical seismic load coefficient" agrees with common pseudostatic notions regarding the onset of uplifting : Uplift is more likely, i. e. $\phi_{c r}$ is smaller, for structures with higher aspect ratio, larger top mass ratio, smaller base mass ratio, or thinner stratum of compliant soil. For example systems A and B, 
$\phi_{c r}=0.7$; it is $\phi_{c r}=0.5$ for $\mathrm{C}$ and $\mathrm{D}$.

The "critical" value obtained from Eq. 9 above might be used approximately by comparing with $\phi_{s}$-value computed according to the $d_{s}$-value (spectral deformation) obtained from the response spectra of the particular ground motion, considering only full-contact structure-soil interaction. When the value obtained from Eq. 10 below exceeds $\phi_{c r}$, partial uplift may perhaps be expected, even without an actual time history response analysis. This approximate uplift indicator is to be tested in the examples in this paper.

$$
\phi_{s}=\frac{3(E / m) \cdot\left(I / L^{4}\right) d_{s}}{(1 / 2+a) g}
$$

\section{INPUT GROUND ACCELERATIONS}

A commonly used measure of severity of ground shaking is peak acceleration, hence both the artificial pulse-type ground motion shown in Fig. 7 and El Centro 1940 accelerograms (S $00 \mathrm{E}$ and vertical) are normalized to a peak of $1.0 \mathrm{~g}$ in the horizontal direction and $0.67 \mathrm{~g}$ in the vertical before they are used as inputs. However, other measures of severity of ground motion may be more relevant to the present problem. Psycharis ${ }^{5)}$, for example, reckoning that the size of a dominant pulse is the relevant measure of uplift potential, used the Pacoima Dam $1971 \mathrm{~S} 16 \mathrm{E}$ ground acceleration as input. Elsewhere it was quoted that the size of the biggest pulse of this record is almost three times as large as the biggest pulse of the El Centro $1940 \mathrm{~S} 00 \mathrm{E}$ record, which is $0.525 \mathrm{~m} / \mathrm{sec}$.

The biggest pulse of the present normalized El Centro record is already about $1.64 \mathrm{~m} / \mathrm{sec}$ ( 3.12 times the actual, nonnormalized), but in order to highlight

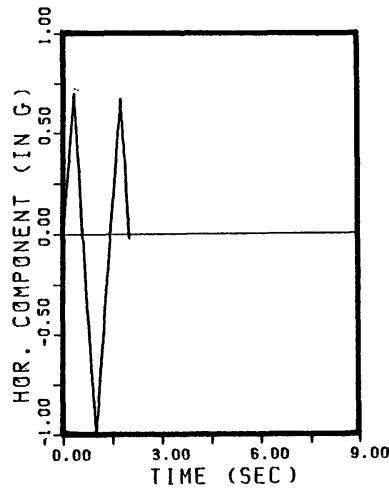

(a)

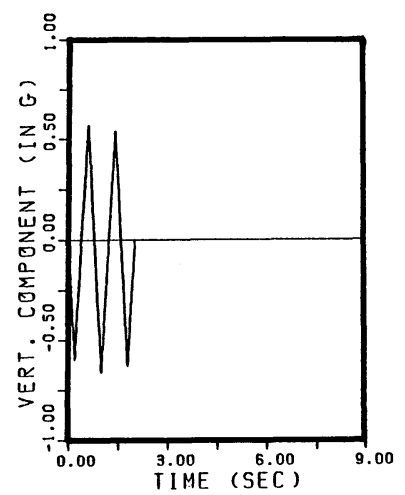

(b)

Fig. 7 Artificial pulse-type ground acceleration in horizontal (a) and vertical (b) directions.

Table 3 Minimum values of normalized effective radius $\left(R^{\prime} / R\right)$ during response.

\begin{tabular}{|c|c|c|c|c|}
\hline System & A & B & C & D \\
\hline Normalized El Centro 1940 & $1.00 *$ & 0.97 & $1.00^{\star}$ & 0.85 \\
\hline Artificial Pulse & 0.84 & ---- & 0.84 &.-- \\
\hline
\end{tabular}

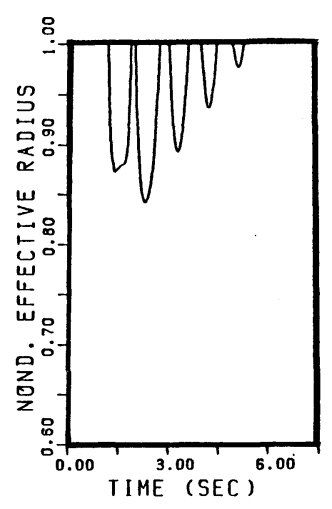

(a)

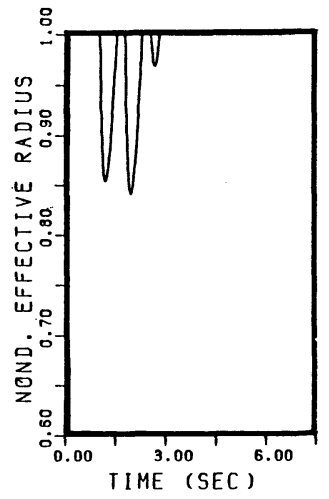

(b)

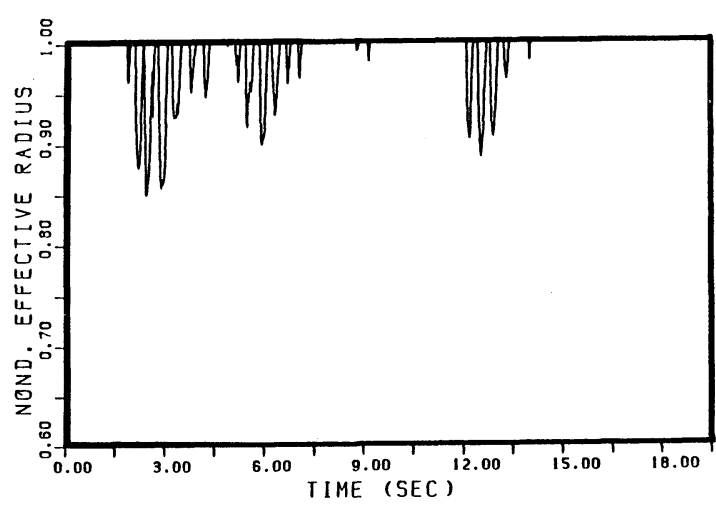

(c)

Fig. 8 Time histories of normalized effective radius $\left(R^{\prime} / R\right)$ for system A (a) and system $\mathrm{C}(\mathrm{b})$ subject to pulse-type acceleration, and/system D (c) subject to normalized El Centro acceleration. 


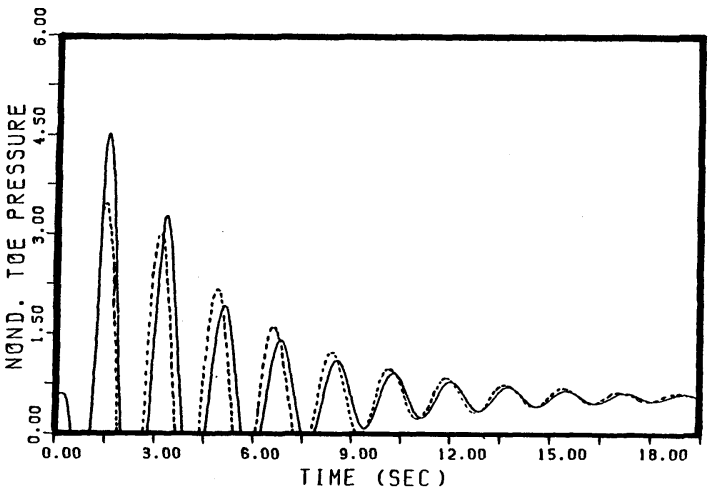

(a)

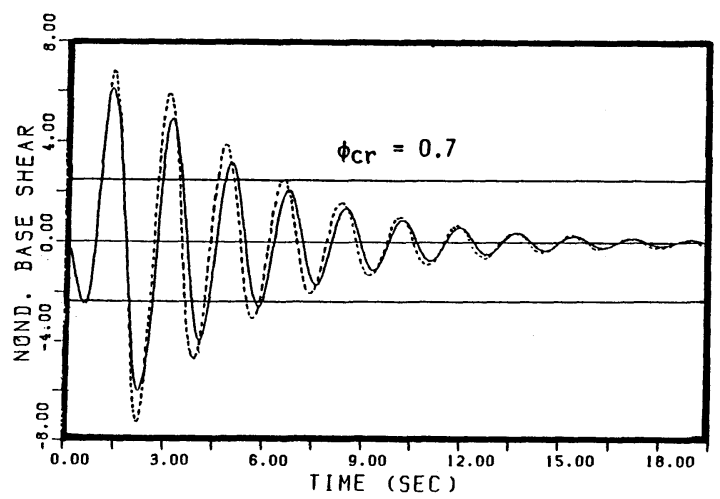

(b)

Fig. 9 Normalized toe pressure (a) and base shear as $V / V_{0}$ (b) for system A subject to pulse-type input. Corresponding Values when uplift is disregarded are shown in dotted curves.

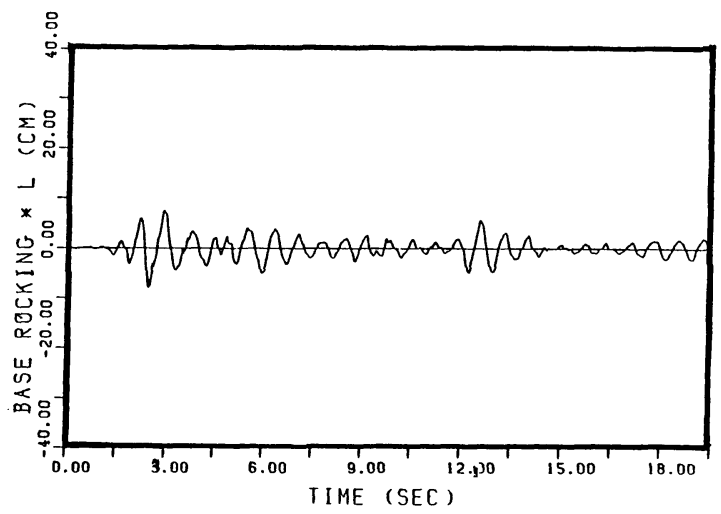

(a)

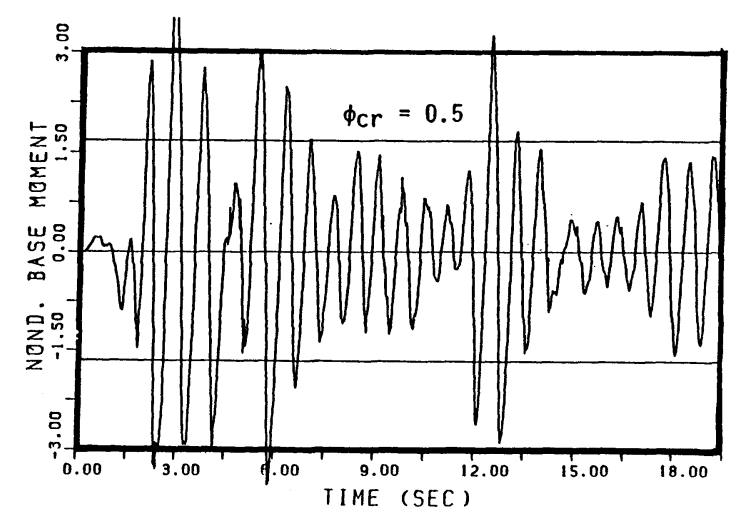

(b)

Fig. 10 Tip displacement due to rigid-body rocking (a) and normalized base/moment as $M / M_{0}$ (b) for system D subject to normalized El Centro input. Also shown (b) is the level corresponding to $\phi_{c r}$ (Eq.8).

the effect of large pulses, the accelerograms of Fig. 7 are also made up such that the big pulse in the horizontal direction is $4.06 \mathrm{~m} / \mathrm{sec}$ and the two smaller ones are half as big. The respective durations of the small and big pulses are $0.59 \mathrm{sec}$ and $0.83 \mathrm{sec}$, comparable to the $0.65 \mathrm{sec}$ duration of the biggest pulse of the Pacoima Dam record.

\section{EFFECT OF UPLIFT ON RESPONSE}

Table 3 shows that of the six seismic responses being considered, only three involve significant uplifting. The time histories of effective radii for these cases are shown in Fig. 8.

Fig. 9 shows two response variables for system A subject to the pulse motion. The dotted lines represent the calculated response when uplift is neglected, i. e. using the usual constant-contact assumption. It is obvious from the curves that uplifting causes an elongated apparent natural period. Also, the constant-contact assumption overestimates base shear and underestimates toe pressure.

Shown in Fig. 10 are two different response variables for system D subject to the normalized El Centro motion. The

Table 4 Over (+) or underestimate $(-)$ of calculated response when uplift is disregarded.

\begin{tabular}{|lccc|}
\hline \multicolumn{1}{|c}{ System } & A & \multicolumn{1}{c|}{ C } & D \\
\hline \multicolumn{1}{|c}{ Input } & PULSE & PULSE & EL CENTRO \\
\hline Top shear force & $25 \%$ & 2 & 2 \\
Base shear force & 20 & 5 & 15 \\
Base moment & 21 & 5 & 13 \\
Toe pressure & -22 & -17 & -21 \\
Rocking & -24 & -22 & -25 \\
\hline
\end{tabular}


base moment is indeed very nearly proportional to the base shear (not shown), but the ratio is about $0.808 \mathrm{~L}$ (instead of $1.0 \mathrm{~L}$ as would be in SDOF oscillator model) in the case of system D. (It is $0.734 \mathrm{~L}$ in system A). As for the base rocking, which is increased by uplifting, the response shown is actually the rocking angle in radians multiplied by the structure height, i. e. tip displacement due to rigid-body rotation; hence of two otherwise identical structures the taller one will rotate less. Table 4 summarizes the effect of neglecting partial uplift. The discrepancies appear to be significant.

\section{A REVIEW OF UPLIFT INDICATORS}

The pulse-type ground acceleration triggers uplift in systems $\mathrm{A}$ and $\mathrm{C}$ while the normalized El Centro does not. The uplifting is larger in $A$ than in $C$, and so are the other response variables (not shown). Two factors may explain why $A$ responds more : the apparent natural frequency of $A$ is closer to a peak in the power spectrum of the input than is $\mathrm{C}$; and the damping in $\mathrm{A}$ is less.

Should the acceleration values (hence the pulses) of the artificial pulse-type ground motion be reduced to $40 \%$ (or peak horizontal acceleration of $0.4 \mathrm{~g}$ ) the maximum pulse will be about equal to the maximum pulse of the normalized El Centro record. That miniature pulse-type ground motion can be shown to be incapable of uplifting A or C. Likewise, as Table 3 confirms, the normalized El Centro ground motion is insufficient.

The normalized El Centro input is sufficient, however, to cause small uplift in system B and significant uplift in D. It should be noted that B and D are identical to A and C, respectively, except for the smaller structure-soil interaction, i. e. less period elongation and less damping increase. In this connection it may be noted that it is also understandable that as the ground becomes more rigid the tendency of structures to uplift increases - and so does the corresponding amount of uplift. Indeed when the ground is rigid, even relatively low-frequency structures may uplift.

Going back to the approximate uplift indicator of Eq.9, the spectral value of $\phi_{s}$, corresponding to the normalized El Centro input may be obtained from Eq. 10 using natural frequency and damping ratio at full-contact interaction condition. The results would be about $\phi_{s}=0.5,0.5,2.0$ and 2.4 for $\mathrm{A}, \mathrm{B}, \mathrm{C}$ and $\mathrm{D}$, respectively. Comparing these with the corresponding "critical" values $\phi_{c r}=0.7$ and $\phi_{c r}=0.5$, one might expect both $\mathrm{C}$ and $\mathrm{D}$ to uplift and both $\mathrm{A}$ and $\mathrm{B}$ not to uplift. However, as herein demonstrated, the actual dynamic response is different, as $B$, rather than $C$, uplifts together with $D$.

Each case here of actual uplift very closely satisfies Eq. 9 itself, though, as may be seen in Figs. 9 (b) and $10(b)$, where the nondimensionalized forces exceed $\phi_{c r} / \phi=0.7 / 0.3=2.3$ (for system A) or $0.5 / 0.3$ $=1.7$ (for D).

\section{SUMMARY AND CONCLUSION}

This study leads to the following remarks and conclusions:

(1) When an above-ground vertical structure is subjected to large ground accelerations, the base may partly uplift unless prevented by design. It may be more economical to allow, anticipate, and design for this condition of uplifting.

(2) Compared to the case when uplifting is prevented, actual uplift reduces structural stresses but increases rigid-body displacements and soil pressures.

(3) The triggering of uplift is a dynamic phenomenon, hence dependent on the details of input ground motion, in particular on the presence of large pulses. The pseudostatic test of comparing $\phi_{c r}$ and $\phi_{s}$ for example (Eqs.9-10), cannot accurately predict the onset of uplifting.

(4) Uplift itself, when it occurs, is nonlinear and transient in nature. It may be amplified by any near-resonance between structure-soil fundamental frequency and power-rich frequencies of the input motion. As both full-contact interaction and partial uplifting reduce the structure-soil apparent frequency, input ground motions whose response spectra are high in the low-frequency range of, say, $0.5-1.0 \mathrm{hz}$ 
become very relevant.

(5) A relatively simple modeling such as the one described here may be an economical tool for a dynamic analysis of uplifting of a number of superstructure types, under varying conditions of structure-soil interaction, and subject to ground accelerations of different characteristics. However, this model neglects sliding and assumes that shear keys may be effective in preventing it in the global level. Studies like Ref. 1 demonstrate that local sliding may be significant in the case of squat structures.

\section{Appendix}

Equations of Motion

$$
\begin{aligned}
& {\left[M^{*}\right]\{\ddot{q}\}+\sqrt{\frac{E I}{m L^{4}}}\left[C^{*}\right]\{\ddot{q}\}+\frac{E I}{m L^{4}}\left[K^{*}\right]\{q\}=-\left\{F^{*}\right\}} \\
& \{q\}=\left\langle\begin{array}{lllll}
d & \alpha L & u & \theta L & v\rangle^{T}
\end{array}\right. \\
& \left\{F^{*}\right\}=\left\langle(a-1 / 2) \ddot{u}_{g} \quad(-1 / 12) \ddot{u}_{g} \quad(a+b+1) \ddot{u}_{g} \quad(a+1 / 2) \ddot{u}_{g} \quad(a+b+1) \ddot{v}_{g}\right\rangle^{T} \text {, } \\
& {\left[K^{*}\right]=\left[\begin{array}{ccccc}
12 & -6 & 0 & 0 & 0 \\
& 4 & 0 & 0 & 0 \\
& & k_{u}^{*} & 0 & 0 \\
& & k_{\theta}^{*} & 0 \\
\text { symmetric } & & k_{v}^{*}
\end{array}\right]-\gamma\left[\begin{array}{ccccc}
(36+72 a) & (-6-6 a) & 0 & (30+60 a) & 0 \\
& (2+8 a) & 0 & (-5) & 0 \\
& & 0 & 0 & 0 \\
\text { symmetric } & & & (30+60 a) & 0 \\
& & & &
\end{array}\right]} \\
& {\left[C^{*}\right]=\left[\begin{array}{ccccc}
0 & 0 & 0 & 0 & 0 \\
& 0 & 0 & 0 & 0 \\
& & c_{u}^{*} & 0 & 0 \\
& & c_{\theta}^{*} & 0 \\
\text { symmetric } & c_{v}^{*}
\end{array}\right]+\left.\frac{2 \zeta}{\left.\omega_{1}\right|_{\ddot{v}_{g=0, R^{\prime}=R}}}\left[K^{*}\right]\right|_{\ddot{v}_{g=0, R^{\prime}=R}}}
\end{aligned}
$$

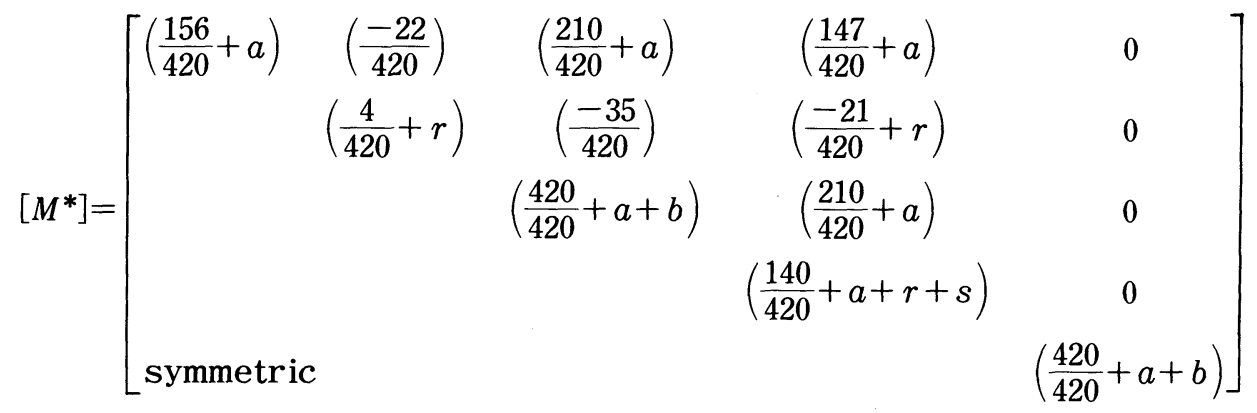

where :

$$
\begin{aligned}
& k_{u}^{*}=\frac{8\left[1+(1 / 2)\left(R^{\prime} / H\right)\right] k_{u}}{[2-\nu][2+2 \nu]\left(E / E_{g}\right)\left(I / L^{4}\right)(L / R)\left(R / R^{\prime}\right)} \\
& k_{\theta}^{*}=\frac{8\left[1+(1 / 6)\left(R^{\prime} / H\right)\right] k_{\theta}}{[3-3 \nu][2+2 \nu]\left(E / E_{g}\right)\left(I / L^{4}\right)(L / R)^{3}\left(R / R^{\prime}\right)^{3}} \\
& k_{v}^{*}=\frac{4\left[1+1.28\left(R^{\prime} / H\right)\right] k_{v}}{[1-\nu][2+2 \nu]\left(E / E_{g}\right)\left(I / L^{4}\right)(L / R)\left(R / R^{\prime}\right)} \\
& c_{u}^{*}=\frac{8\left[1+(1 / 2)\left(R^{\prime} / H\right)\right] c_{u}}{\pi[2-\nu] \sqrt{2+2 \nu} \sqrt{m / \pi R^{2} \rho} \sqrt{I / L^{4}}(L / R)\left(R / R^{\prime}\right)^{2}} \\
& c_{\theta}^{*}=\frac{8\left[1+(1 / 6)\left(R^{\prime} / H\right)\right] c_{\theta}}{\pi[3-3 \nu] \sqrt{2+2 \nu} \sqrt{m / \pi R^{2} \rho} \sqrt{I / L^{4}}(L / R)^{3}\left(R / R^{\prime}\right)^{4}} \\
& c_{v}^{*}=\frac{4\left[1+1.28\left(R^{\prime} / H\right)\right] c_{v}}{\pi[1-\nu] \sqrt{2+2 \nu} \sqrt{m / \pi R^{2} \rho} \sqrt{I / L^{4}}(L / R)\left(R / R^{\prime}\right)^{2}}
\end{aligned}
$$




$$
\begin{aligned}
& \gamma=\gamma(t)=\frac{(\pi / 60)\left(m / \pi R^{2} \rho\right)\left(\rho g R / E_{g}\right)\left(1+\ddot{v}_{g} / g\right)}{\left(E / E_{g}\right)\left(I / L^{4}\right)(L / R)} \\
& k_{j}=k_{j}\left(a_{0}, \nu, \xi, R^{\prime} / H\right)=\text { stiffness coefficient in base mode } j \\
& c_{j}=c_{j}\left(a_{0}, \nu, \xi, R^{\prime} / H\right)=\text { damping coefficient in base node } j
\end{aligned}
$$

\section{REFERENCES}

1) Toki, K., Sato, T. and Miura, F. : Separation and Sliding between Soil and Structure During Strong Ground Motion, Earthquake Eng. Struct. Dyn., Vol.9, pp. 263 277, 1981.

2) Desai, C. and Zaman, M. : Influence of Interface Behaviour in Dynamic Soil-Structure Interaction, Proc. 8th World Conf. Earthquake Eng., III, pp. 1049 1056, 1984.

3) Yim, C. -S. and Chopra, A. : Earthquake Response of Structures with Partial Uplift on Winkler Foundation, Earthquake Eng. Struct. Dyn., Vol. 12, pp. 263 281, 1984.

4) Chopra, A. and Yim, C.-S. : Simplified Analysis of Structures with Foundation Uplift, J. Struct. Div., ASCE, Vol.111, No. 4, pp. 906 930, 1985.

5) Psycharis, 1. : Dynamics of Flexible Systems with Partial Uplift, Earthquake Eng. Struct. Dyn., Vol. 11, pp. 501 521, 1983.

6) Fukuzawa, R., Chiba, O., Hatori, T. and Tohdo, M. : Rocking Vibration of Nuclear Power Plant Considering Uplift and Yield of Supporting Soil, Sixth Int. Conf. Struct. Mech. Reactor Tech., Paper K 3/7, 1981.

7) Wolf, J. and Skrikerud, P. : Seismic Excitation with Large Overturning Moment : Tensile Capacity, Projecting Base Mat or Lifting-off?, Nuclear Eng. Design, Vol.50, pp. 305 321, 1978.

8) Takemori, T., Sotomura, K. and Yamada, M. : Nonlinear Dynamic Response of Reactor Containment, Nuclear Eng. Design, Vol. 38, pp. 463 474, 1976 .

9) Kennedy, R., Short, S., Wesley, D. and Lee, T. : Effects on Non-linear Soil-Structure Interaction due to Base Slab Uplift, Nuclear Eng. Design, Vol. 38, pp. 323 355, 1976.

10) Veletsos, A. and Verbic, B. : Vibration of Viscoelastic Foundations, Earthquake Eng. Struct. Dyn., Vol.2, pp. 87 102, 1973.

11) Kausel, E. and Roesset, J. : Dynamic Stiffness of Circular Foundations, J. Eng. Mech. Div., ASCE, Vol. 101, No.6, pp.771 $\sim 785,1975$.

12) Ghaffar-Zadeh, M. and Chapel, F. : Frequency-independent Impedances of Soil-Structure Systems in Horizontal and Rocking Modes, Earthquake Eng. Struct. Dyn., Vol.11, pp. 523 540, 1983.

13) Hurty, W. and Rubinstein, M. : Dynamics of Structures, Prentice-Hall, pp. 299 307, 1964.

(Received December 16 1985) 